Supporting Information

\title{
The Influence of Molecular Architecture and Chain Flexibility on the Phase Map of Polystyrene-block- Poly(dimethylsiloxane) Brush Block Copolymers
}

Hua-Feng Fei, Benjamin M. Yavitt, Xiyu Hu, Gayathri Kopanati, Alexander Ribbe and James J. Watkins*

Department of Polymer Science and Engineering, University of Massachusetts Amherst, 120 Governors Drive, Amherst MA 01003

\section{Materials}

2-Hydroxyethyl 2-Bromoisobutyrate (>99.0), Exo-5-norbornenecarboxylic acid (>97\%), dicyclohexyl-carbodiimide (DCC, >99.0\%), N,N,N',N', N',-Pentamethyldiethylenetriamine (PMDETA, 99\%), N,N'- 4-dimethylaminopyridine (DMAP, >99\%), Triethylamine (TEA, 99.5\%) and Grubbs $3^{\text {rd }}$ generation catalyst were purchased from Sigma-Aldrich. Anhydrous tetrahydrofuran (THF, 99.9\%) and dichloromethane (DCM, 99.9\%) were purchased from Acros Organics. All of the listed chemicals were used without further purification. Styrene was purchased from Sigma-Aldrich and passed through basic aluminum oxide before controlled polymerization. Hydroxy terminated polydimethylsiloxane (PDMS-OH, $M_{n}=4670 \mathrm{~g} / \mathrm{mol}$ ) was purchased from Sigma-Aldrich and heated at $120^{\circ} \mathrm{C}$ for $4 \mathrm{~h}$ under nitrogen flow to remove moisture before use.

\section{Samples preparation and characterization}

Gel permeation chromatography (GPC) of the bottlebrush block copolymers was carried out in THF +1 vol. $\%$ Triethylamine (TEA) on two PLgel $10 \mu \mathrm{m}$ mixed-B LS columns (Polymer Laboratories) connected in series with a Wyatt Technologies DAWN EOS multi-angle laser light 
scattering (MALLS) detector and RI detector. No calibration standards were used for the bottlebrush block copolymers. $d n / d c$ values were obtained for each injection by assuming $100 \%$ mass elution from the columns. GPC of macromonomers was carried out using a Polymer Laboratories PL-GPC50 instrument with two $5 \mu \mathrm{m}$ mixed-D columns, a $5 \mu \mathrm{m}$ guard column, and a RI detector (HP1047A). THF was used as the eluent at a flow rate of $1.0 \mathrm{~mL} / \mathrm{min}$. Polystyrene standards were used for the calibration. Proton nuclear magnetic resonance $\left({ }^{1} \mathrm{H}\right.$ NMR) spectroscopy was measured in $\mathrm{CDCl}_{3}$ using a Bruker 500 NMR spectrometer. Small-angle X-ray scattering (SAXS) measurements were conducted at Beamline 11-BM of the National Synchrotron Light Source-II (Brookhaven National Lab). Bulk BBCP samples were sealed into a circular mold assembly with Kapton tape and secured to the sample stage in transmission geometry. The measurement exposure time was set to 15 seconds. Transmission electron microscopy (TEM) measurements were conducted with a JEOL 2000FX TEM operated at an accelerating voltage of $200 \mathrm{kV}$. Thin sections of approximately $50 \mathrm{~nm}$ in thickness were prepared using a Leica Ultracut UCT microtome equipped with a Leica EM FCS cryogenic sample S2 chamber operated at $-140{ }^{\circ} \mathrm{C}$. The thin cryo-microtomed sections were left unstained as contrast arises from the natural differences in nuclear mass present in PS (light domains) and PDMS (dark domains) side chains. Scanning transmission electron microscopy in high angle annular dark field imaging mode (STEM-HAADF) tomography was carried out using a JEOL JEM-2200FS at $200 \mathrm{kV}$ acceleration voltage and a probe size of $1.5 \mathrm{~nm}$. Image series of 101 images for tomography reconstruction were recorded at $1^{\circ}$ steps from $-50^{\circ}$ to $+50^{\circ}$ tilt angles. Three-dimensional reconstructions were generated using the filtered back projection algorithm in Etomo (part of the IMOD software package, UC Boulder). Volume and iso surface rendering were performed using Chimera software (UCSF). 


\section{Synthesis of macromonomers and brush block copolymers}

All the synthesis procedures followed those discussed in previous literature. ${ }^{1}$ The volume fraction of each sample was calculated assuming densities of PS $(1.05 \mathrm{~g} / \mathrm{mL})$ and PDMS $(0.96 \mathrm{~g} / \mathrm{mL})$.

Table S1 Macromonomer (MM) Molecular Characteristics

\begin{tabular}{|c|c|c|c|c|}
\hline MM Name & $M_{n}(\mathrm{~kg} / \mathrm{mol})^{\mathrm{a}}$ & $M_{n}(\mathrm{~kg} / \mathrm{mol})^{\mathrm{b}}$ & Dispersity (Đ) ${ }^{b}$ & $N_{s c} \mathrm{c}$ \\
\hline PDMS-NB & 4.8 & 5.0 & 1.10 & 65 \\
\hline PS-NB (3.1k) & 3.1 & 3.0 & 1.05 & 29 \\
\hline PS-NB (4.7k) & 4.7 & 4.7 & 1.07 & 45 \\
\hline PS-NB (7.6k) & 7.6 & 7.6 & 1.12 & 73 \\
\hline
\end{tabular}

${ }^{\mathrm{a}} M_{n}$ was measured by ${ }^{1} \mathrm{HMR} .{ }^{\mathrm{b}} M_{n}$ and $\mathrm{Ð}$ were determined by GPC. ${ }^{\mathrm{c}}$ Macromonomer degree of polymerization (equivalent to side chain degree of polymerization, $N_{s c}$ ) were calculated using molecular weights as measured by ${ }^{1} \mathrm{NMR}$.

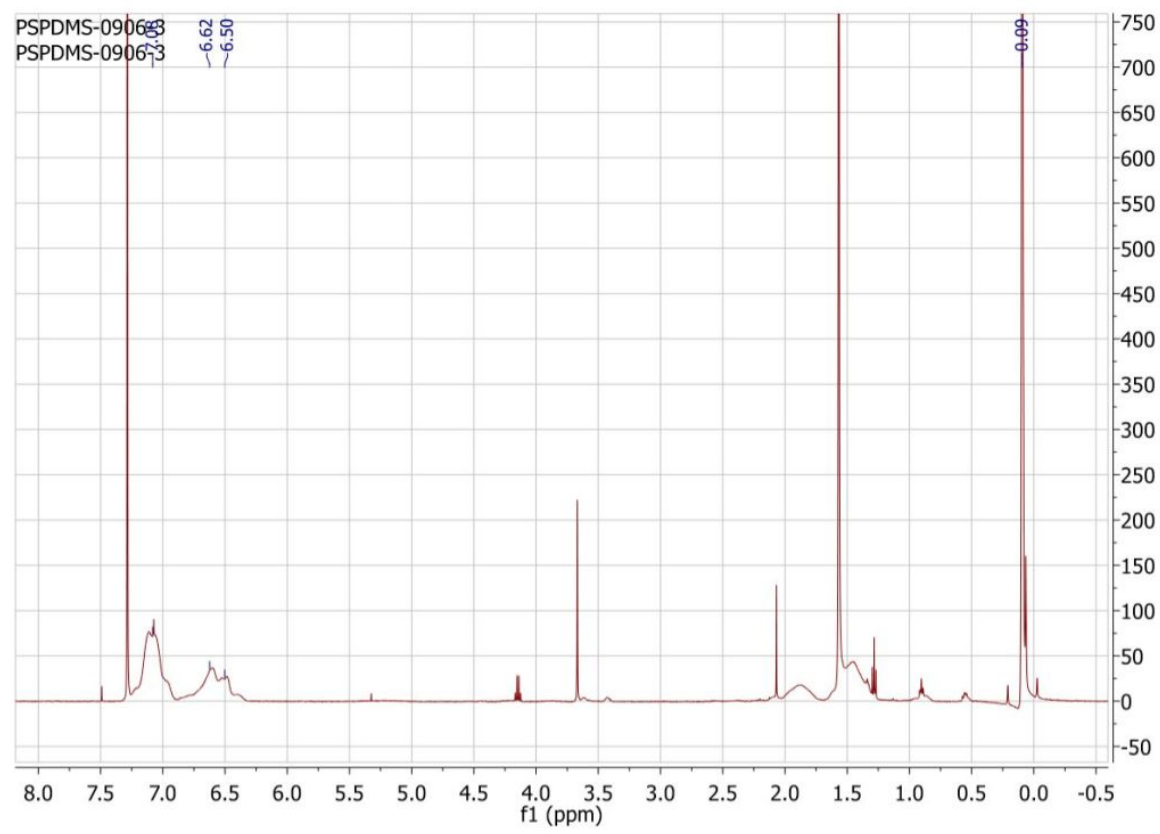

Figure S1 ${ }^{1} \mathrm{H}$ NMR spectra of representative PS- $b$-PDMS brush block copolymer. 


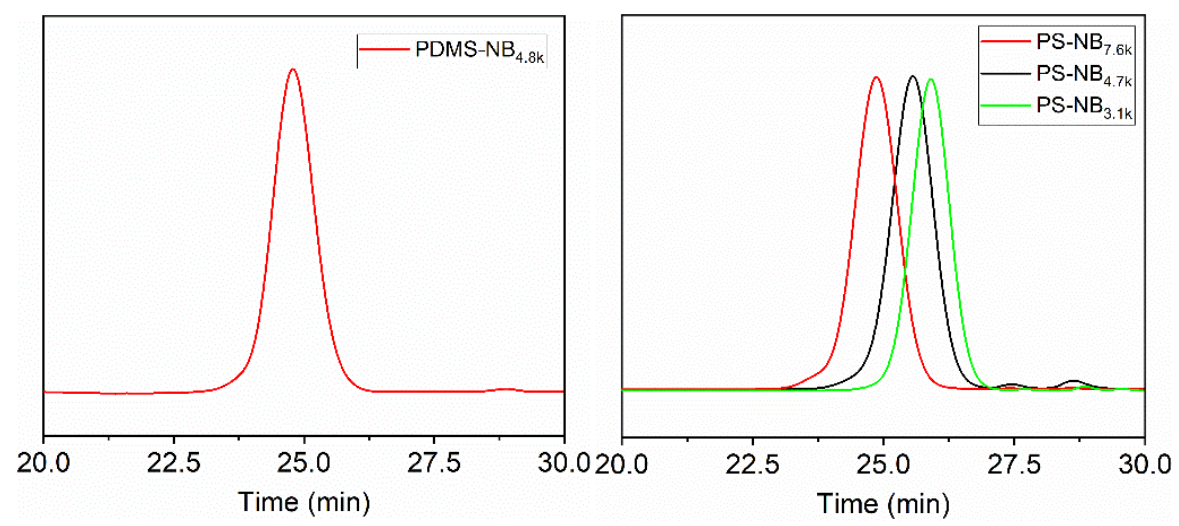

Figure S2 GPC traces of all macromonomers (PDMS-NB and PS-NB).
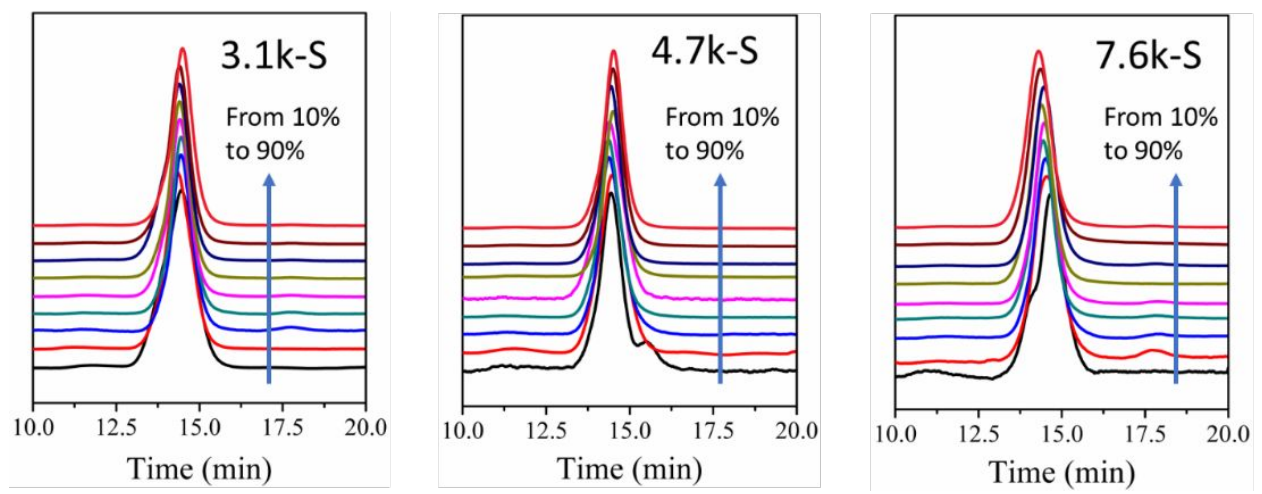

Figure S3 GPC MALLS traces of short backbone length BBCPs grouped by PS side chain length
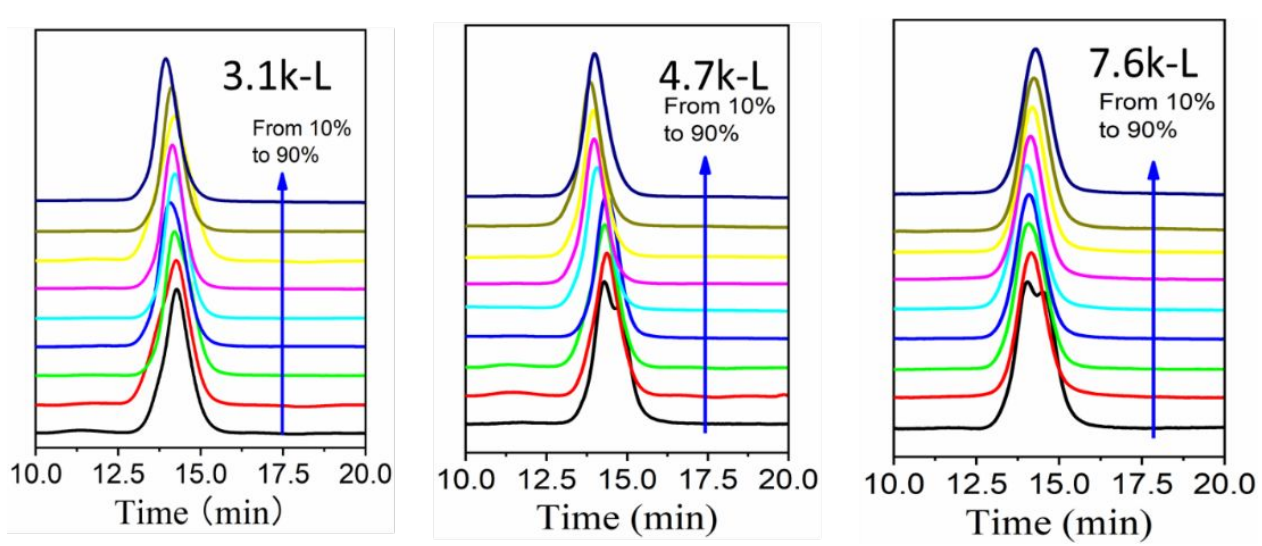

Figure S4 GPC MALLS traces of long backbone length BBCPs grouped by PS side chain length 
Table S2 Detailed molecular information of all BBCPs samples

\begin{tabular}{|c|c|c|c|c|c|c|c|c|}
\hline Sample code & $d n / d c$ & $\begin{array}{c}M \mathbf{M w} / \mathbf{1 0} \mathbf{3}^{3} \\
\mathrm{~g} / \mathrm{mol}\end{array}$ & $\begin{array}{c}M \mathrm{Mn} / 10^{3} \\
\mathrm{~g} / \mathrm{mol}\end{array}$ & PDI & $f_{P S}$ & $N_{b b}(\mathrm{PS})$ & $N_{b b}($ PDMS $)$ & $N_{b b}($ total $)$ \\
\hline $3.1 \mathrm{k}-\mathrm{S}-90 \% \mathrm{PS}$ & 0.131 & 236 & 219 & 1.08 & $89.2 \%$ & 63 & 5 & 68 \\
\hline $3.1 \mathrm{k}-\mathrm{S}-80 \% \mathrm{PS}$ & 0.115 & 268 & 233 & 1.15 & $78.5 \%$ & 60 & 10 & 70 \\
\hline $3.1 \mathrm{k}-\mathrm{S}-70 \% \mathrm{PS}$ & 0.098 & 260 & 232 & 1.12 & $68.1 \%$ & 52 & 15 & 67 \\
\hline $3.1 \mathrm{k}-\mathrm{S}-60 \% \mathrm{PS}$ & 0.090 & 272 & 237 & 1.15 & $57.8 \%$ & 46 & 20 & 66 \\
\hline $3.1 \mathrm{k}-\mathrm{S}-50 \% \mathrm{PS}$ & 0.075 & 269 & 238 & 1.13 & $47.8 \%$ & 38 & 25 & 63 \\
\hline $3.1 \mathrm{k}-\mathrm{S}-40 \% \mathrm{PS}$ & 0.062 & 250 & 229 & 1.09 & $37.9 \%$ & 30 & 30 & 60 \\
\hline $3.1 \mathrm{k}-\mathrm{S}-30 \% \mathrm{PS}$ & 0.055 & 254 & 233 & 1.09 & $28.2 \%$ & 22 & 34 & 56 \\
\hline $3.1 \mathrm{k}-\mathrm{S}-20 \% \mathrm{PS}$ & 0.038 & 342 & 285 & 1.20 & $18.6 \%$ & 18 & 48 & 66 \\
\hline $3.1 \mathrm{k}-\mathrm{S}-10 \% \mathrm{PS}$ & 0.030 & 335 & 275 & 1.22 & $9.2 \%$ & 9 & 52 & 61 \\
\hline $3.1 \mathrm{k}-\mathrm{L}-90 \% \mathrm{PS}$ & 0.126 & 775 & 635 & 1.22 & $89.2 \%$ & 184 & 13 & 197 \\
\hline $3.1 \mathrm{k}-\mathrm{L}-80 \% \mathrm{PS}$ & 0.115 & 617 & 532 & 1.16 & $78.5 \%$ & 137 & 22 & 159 \\
\hline $3.1 \mathrm{k}-\mathrm{L}-70 \% \mathrm{PS}$ & 0.101 & 632 & 527 & 1.20 & $68.1 \%$ & 119 & 33 & 152 \\
\hline $3.1 \mathrm{k}-\mathrm{L}-60 \% \mathrm{PS}$ & 0.089 & 659 & 558 & 1.18 & $57.8 \%$ & 108 & 47 & 155 \\
\hline $3.1 \mathrm{k}-\mathrm{L}-50 \% \mathrm{PS}$ & 0.070 & 678 & 589 & 1.15 & $47.8 \%$ & 95 & 61 & 156 \\
\hline $3.1 \mathrm{k}-\mathrm{L}-40 \% \mathrm{PS}$ & 0.067 & 713 & 609 & 1.17 & $37.9 \%$ & 78 & 76 & 154 \\
\hline $3.1 \mathrm{k}-\mathrm{L}-30 \% \mathrm{PS}$ & 0.057 & 589 & 471 & 1.25 & $28.2 \%$ & 45 & 69 & 114 \\
\hline $3.1 \mathrm{k}-\mathrm{L}-20 \% \mathrm{PS}$ & 0.043 & 586 & 469 & 1.25 & $18.6 \%$ & 30 & 78 & 108 \\
\hline $3.1 \mathrm{k}-\mathrm{L}-10 \% \mathrm{PS}$ & 0.030 & 580 & 457 & 1.27 & $9.2 \%$ & 15 & 86 & 101 \\
\hline $4.7 \mathrm{k}-\mathrm{S}-90 \% \mathrm{PS}$ & 0.133 & 380 & 349 & 1.09 & $89.2 \%$ & 67 & 7 & 74 \\
\hline $4.7 \mathrm{k}-\mathrm{S}-80 \% \mathrm{PS}$ & 0.124 & 372 & 335 & 1.11 & $78.5 \%$ & 57 & 14 & 71 \\
\hline $4.7 \mathrm{k}-\mathrm{S}-70 \% \mathrm{PS}$ & 0.103 & 395 & 359 & 1.10 & $68.1 \%$ & 53 & 22 & 75 \\
\hline $4.7 \mathrm{k}-\mathrm{S}-60 \% \mathrm{PS}$ & 0.085 & 330 & 300 & 1.10 & $57.8 \%$ & 38 & 25 & 63 \\
\hline $4.7 \mathrm{k}-\mathrm{S}-50 \% \mathrm{PS}$ & 0.080 & 346 & 320 & 1.08 & $47.8 \%$ & 34 & 33 & 67 \\
\hline $4.7 \mathrm{k}-\mathrm{S}-40 \% \mathrm{PS}$ & 0.075 & 341 & 313 & 1.09 & $37.9 \%$ & 26 & 39 & 65 \\
\hline $4.7 \mathrm{k}-\mathrm{S}-30 \% \mathrm{PS}$ & 0.058 & 345 & 308 & 1.12 & $28.2 \%$ & 19 & 46 & 65 \\
\hline $4.7 \mathrm{k}-\mathrm{S}-20 \% \mathrm{PS}$ & 0.048 & 335 & 286 & 1.17 & $18.6 \%$ & 12 & 47 & 59 \\
\hline $4.7 \mathrm{k}-\mathrm{S}-10 \% \mathrm{PS}$ & 0.028 & 373 & 308 & 1.21 & $9.2 \%$ & 6 & 58 & 64 \\
\hline $4.7 \mathrm{k}-\mathrm{L}-90 \% \mathrm{PS}$ & 0.130 & 639 & 492 & 1.30 & $89.2 \%$ & 94 & 11 & 105 \\
\hline $4.7 \mathrm{k}-\mathrm{L}-80 \% \mathrm{PS}$ & 0.109 & 654 & 507 & 1.29 & $78.5 \%$ & 86 & 21 & 107 \\
\hline $4.7 \mathrm{k}-\mathrm{L}-70 \% \mathrm{PS}$ & 0.101 & 642 & 518 & 1.24 & $68.1 \%$ & 77 & 32 & 109 \\
\hline $4.7 \mathrm{k}-\mathrm{L}-60 \% \mathrm{PS}$ & 0.090 & 687 & 550 & 1.25 & $57.8 \%$ & 70 & 46 & 116 \\
\hline $4.7 \mathrm{k}-\mathrm{L}-50 \% \mathrm{PS}$ & 0.075 & 684 & 570 & 1.20 & $47.8 \%$ & 60 & 59 & 119 \\
\hline $4.7 \mathrm{k}-\mathrm{L}-40 \% \mathrm{PS}$ & 0.063 & 678 & 560 & 1.21 & $37.9 \%$ & 48 & 70 & 118 \\
\hline $4.7 \mathrm{k}-\mathrm{L}-30 \% \mathrm{PS}$ & 0.046 & 632 & 527 & 1.20 & $28.2 \%$ & 34 & 77 & 111 \\
\hline $4.7 \mathrm{k}-\mathrm{L}-20 \% \mathrm{PS}$ & 0.035 & 596 & 458 & 1.30 & $18.6 \%$ & 19 & 76 & 95 \\
\hline $4.7 \mathrm{k}-\mathrm{L}-10 \% \mathrm{PS}$ & 0.027 & 556 & 445 & 1.25 & $9.2 \%$ & 10 & 83 & 93 \\
\hline $7.6 \mathrm{k}-\mathrm{S}-90 \% \mathrm{PS}$ & 0.136 & 380 & 352 & 1.08 & $89.2 \%$ & 42 & 7 & 49 \\
\hline $7.6 \mathrm{k}-\mathrm{S}-80 \% \mathrm{PS}$ & 0.123 & 360 & 327 & 1.10 & $78.5 \%$ & 34 & 14 & 48 \\
\hline $7.6 \mathrm{k}-\mathrm{S}-70 \% \mathrm{PS}$ & 0.105 & 350 & 321 & 1.09 & $68.1 \%$ & 29 & 20 & 49 \\
\hline $7.6 \mathrm{k}-\mathrm{S}-60 \% \mathrm{PS}$ & 0.088 & 363 & 324 & 1.12 & $57.8 \%$ & 25 & 27 & 52 \\
\hline $7.6 \mathrm{k}-\mathrm{S}-50 \% \mathrm{PS}$ & 0.081 & 343 & 301 & 1.14 & $47.8 \%$ & 20 & 31 & 51 \\
\hline $7.6 \mathrm{k}-\mathrm{S}-40 \% \mathrm{PS}$ & 0.072 & 374 & 322 & 1.16 & $37.9 \%$ & 17 & 40 & 57 \\
\hline $7.6 \mathrm{k}-\mathrm{S}-30 \% \mathrm{PS}$ & 0.058 & 369 & 318 & 1.16 & $28.2 \%$ & 12 & 46 & 58 \\
\hline $7.6 \mathrm{k}-\mathrm{S}-20 \% \mathrm{PS}$ & 0.049 & 346 & 288 & 1.20 & $18.6 \%$ & 7 & 48 & 55 \\
\hline $7.6 \mathrm{k}-\mathrm{S}-10 \% \mathrm{PS}$ & 0.032 & 328 & 269 & 1.22 & $9.2 \%$ & 4 & 50 & 54 \\
\hline $7.6 \mathrm{k}-\mathrm{L}-90 \% \mathrm{PS}$ & 0.127 & 623 & 506 & 1.23 & $89.2 \%$ & 60 & 11 & 71 \\
\hline $7.6 \mathrm{k}-\mathrm{L}-80 \% \mathrm{PS}$ & 0.118 & 646 & 552 & 1.17 & $78.5 \%$ & 58 & 23 & 81 \\
\hline 7.6k-L-70\%PS & 0.105 & 698 & 567 & 1.23 & $68.1 \%$ & 52 & 35 & 87 \\
\hline
\end{tabular}




\begin{tabular}{|c|c|c|c|c|c|c|c|c|}
\hline 7.6k-L-60\%PS & 0.093 & 683 & 594 & 1.15 & $57.8 \%$ & 47 & 49 & 86 \\
\hline 7.6k-L-50\%PS & 0.080 & 756 & 657 & 1.15 & $47.8 \%$ & 43 & 68 & 111 \\
\hline 7.6k-L-40\%PS & 0.067 & 798 & 676 & 1.18 & $37.9 \%$ & 35 & 85 & 120 \\
\hline 7.6k-L-30\%PS & 0.050 & 789 & 658 & 1.20 & $28.2 \%$ & 26 & 96 & 122 \\
\hline 7.6k-L-20\%PS & 0.040 & 689 & 551 & 1.25 & $18.6 \%$ & 15 & 92 & 107 \\
\hline 7.6k-L-10\%PS & 0.029 & 608 & 468 & 1.30 & $9.2 \%$ & 6 & 88 & 94 \\
\hline
\end{tabular}

Table S3 Domain spacing $\left(d_{0}=2 \pi / q^{*}\right)$ of all BBCP samples calculated from primary peak position in SAXS

\begin{tabular}{|c|c|c|c|c|c|c|c|c|}
\hline Sample code & $\begin{array}{c}q^{*} \\
\left(\mathrm{~nm}^{-1}\right)\end{array}$ & $\begin{array}{c}\text { d-spacing } \\
\text { (nm) }\end{array}$ & Sample code & $\begin{array}{c}q^{*} \\
\left(\mathrm{~nm}^{-1}\right)\end{array}$ & $\begin{array}{c}\text { d-spacing } \\
\text { (nm) }\end{array}$ & Sample code & $\begin{array}{c}q^{*} \\
\left(\mathrm{~nm}^{-1}\right)\end{array}$ & $\begin{array}{l}\text { d-spacing } \\
\text { (nm) }\end{array}$ \\
\hline $3.1 \mathrm{k}-\mathrm{S}-90 \% \mathrm{PS}$ & 0.201 & 31.2 & $4.7 \mathrm{k}-\mathrm{S}-90 \% \mathrm{PS}$ & 0.135 & 46.5 & 7.6k-S-90\%PS & 0.205 & 30.6 \\
\hline $3.1 \mathrm{k}-\mathrm{S}-80 \% \mathrm{PS}$ & 0.117 & 53.7 & $4.7 \mathrm{k}-\mathrm{S}-80 \% \mathrm{PS}$ & 0.176 & 35.7 & 7.6k-S-80\%PS & 0.165 & 38.1 \\
\hline $3.1 \mathrm{k}-\mathrm{S}-70 \% \mathrm{PS}$ & 0.148 & 42.4 & $4.7 \mathrm{k}-\mathrm{S}-70 \% \mathrm{PS}$ & 0.135 & 46.5 & $7.6 \mathrm{k}-\mathrm{S}-70 \% \mathrm{PS}$ & 0.130 & 48.3 \\
\hline $3.1 \mathrm{k}-\mathrm{S}-60 \% \mathrm{PS}$ & 0.119 & 52.8 & $4.7 \mathrm{k}-\mathrm{S}-60 \% \mathrm{PS}$ & 0.115 & 54.6 & 7.6k-S-60\%PS & 0.106 & 59.2 \\
\hline $3.1 \mathrm{k}-\mathrm{S}-50 \% \mathrm{PS}$ & 0.097 & 64.7 & $4.7 \mathrm{k}-\mathrm{S}-50 \% \mathrm{PS}$ & 0.101 & 62.2 & 7.6k-S-50\%PS & 0.110 & 57.1 \\
\hline $3.1 \mathrm{k}-\mathrm{S}-40 \% \mathrm{PS}$ & 0.120 & 52.3 & $4.7 \mathrm{k}-\mathrm{S}-40 \% \mathrm{PS}$ & 0.108 & 58.1 & 7.6k-S-40\%PS & 0.089 & 70.5 \\
\hline $3.1 \mathrm{k}-\mathrm{S}-30 \% \mathrm{PS}$ & 0.136 & 46.2 & $4.7 \mathrm{k}-\mathrm{S}-30 \% \mathrm{PS}$ & 0.105 & 59.8 & 7.6k-S-30\%PS & 0.105 & 61.5 \\
\hline $3.1 \mathrm{k}-\mathrm{S}-20 \% \mathrm{PS}$ & 0.162 & 38.8 & $4.7 \mathrm{k}-\mathrm{S}-20 \% \mathrm{PS}$ & 0.149 & 42.1 & 7.6k-S-20\%PS & 0.126 & 49.8 \\
\hline $3.1 \mathrm{k}-\mathrm{S}-10 \% \mathrm{PS}$ & 0.208 & 30.2 & $4.7 \mathrm{k}-\mathrm{S}-10 \% \mathrm{PS}$ & 0.169 & 37.2 & 7.6k-S-10\%PS & 0.139 & 45.2 \\
\hline $3.1 \mathrm{k}-\mathrm{L}-90 \% \mathrm{PS}$ & 0.130 & 48.3 & $4.7 \mathrm{k}-\mathrm{L}-90 \% \mathrm{PS}$ & 0.139 & 45.2 & 7.6k-L-90\%PS & 0.170 & 36.9 \\
\hline $3.1 \mathrm{k}-\mathrm{L}-80 \% \mathrm{PS}$ & 0.115 & 54.6 & $4.7 \mathrm{k}-\mathrm{L}-80 \% \mathrm{PS}$ & 0.109 & 57.6 & $7.6 \mathrm{k}-\mathrm{L}-80 \% \mathrm{PS}$ & 0.132 & 47.6 \\
\hline $3.1 \mathrm{k}-\mathrm{L}-70 \% \mathrm{PS}$ & 0.098 & 64.1 & $4.7 \mathrm{k}-\mathrm{L}-70 \% \mathrm{PS}$ & 0.072 & 87.2 & $7.6 \mathrm{k}-\mathrm{L}-70 \% \mathrm{PS}$ & 0.094 & 66.8 \\
\hline $3.1 \mathrm{k}-\mathrm{L}-60 \% \mathrm{PS}$ & 0.062 & 101.3 & $4.7 \mathrm{k}-\mathrm{L}-60 \% \mathrm{PS}$ & 0.061 & 103.0 & 7.6k-L-60\%PS & 0.075 & 83.7 \\
\hline $3.1 \mathrm{k}-\mathrm{L}-50 \% \mathrm{PS}$ & 0.061 & 103.0 & $4.7 \mathrm{k}-\mathrm{L}-50 \% \mathrm{PS}$ & 0.065 & 96.6 & $7.6 \mathrm{k}-\mathrm{L}-50 \% \mathrm{PS}$ & 0.050 & 125.6 \\
\hline $3.1 \mathrm{k}-\mathrm{L}-40 \% \mathrm{PS}$ & 0.054 & 116.3 & $4.7 \mathrm{k}-\mathrm{L}-40 \% \mathrm{PS}$ & 0.069 & 91.0 & $7.6 \mathrm{k}-\mathrm{L}-40 \% \mathrm{PS}$ & 0.054 & 116.3 \\
\hline $3.1 \mathrm{k}-\mathrm{L}-30 \% \mathrm{PS}$ & 0.061 & 103.0 & $4.7 \mathrm{k}-\mathrm{L}-30 \% \mathrm{PS}$ & 0.082 & 76.6 & 7.6k-L-30\%PS & 0.050 & 125.6 \\
\hline $3.1 \mathrm{k}-\mathrm{L}-20 \% \mathrm{PS}$ & 0.075 & 83.7 & $4.7 \mathrm{k}-\mathrm{L}-20 \% \mathrm{PS}$ & 0.084 & 74.8 & $7.6 \mathrm{k}-\mathrm{L}-20 \% \mathrm{PS}$ & 0.057 & 110.2 \\
\hline $3.1 \mathrm{k}-\mathrm{L}-10 \% \mathrm{PS}$ & 0.136 & 46.2 & $4.7 \mathrm{k}-\mathrm{L}-10 \% \mathrm{PS}$ & 0.109 & 57.6 & 7.6k-L-10\%PS & 0.094 & 66.8 \\
\hline
\end{tabular}

Table S4 Comparison of the lamellar domain spacing $\left(d_{0}=2 \pi / q^{*}\right)$ with respect to the spacing predicted by an extended bilayer or interdigitated packing motif. Calculation based on backbone contour length $L=N_{b b}{ }^{*} L_{0}$, where $L_{0}$ is backbone repeat unit length $(0.62 \mathrm{~nm})$.

\begin{tabular}{|c|c|c|c|}
\hline Sample code & $\begin{array}{c}\text { d-spacing } \\
(\mathbf{n m})\end{array}$ & $\mathbf{N}_{\mathbf{b b}}$ (total) & $\begin{array}{c}\text { Bilayer Limit } \\
\boldsymbol{d}_{\boldsymbol{0}}=\mathbf{2} \boldsymbol{L}(\mathbf{n m})\end{array}$ \\
\hline $3.1 \mathrm{k}-\mathrm{S}-70 \% \mathrm{PS}$ & 42.4 & 67 & 83.1 \\
\hline $3.1 \mathrm{k}-\mathrm{S}-60 \% \mathrm{PS}$ & 52.8 & 66 & 81.8 \\
\hline $3.1 \mathrm{k}-\mathrm{S}-50 \% \mathrm{PS}$ & 64.7 & 63 & 78.1 \\
\hline $3.1 \mathrm{k}-\mathrm{S}-40 \% \mathrm{PS}$ & 52.3 & 60 & 74.4 \\
\hline $3.1 \mathrm{k}-\mathrm{L}-50 \% \mathrm{PS}$ & 103.0 & 156 & 193.4 \\
\hline $3.1 \mathrm{k}-\mathrm{L}-40 \% \mathrm{PS}$ & 116.3 & 154 & 191.0 \\
\hline $4.7 \mathrm{k}-\mathrm{S}-60 \% \mathrm{PS}$ & 54.6 & 63 & 78.1 \\
\hline $4.7 \mathrm{k}-\mathrm{S}-50 \% \mathrm{PS}$ & 62.2 & 67 & 83.1 \\
\hline $4.7 \mathrm{k}-\mathrm{S}-40 \% \mathrm{PS}$ & 58.1 & 65 & 80.6 \\
\hline $4.7 \mathrm{k}-\mathrm{L}-60 \% \mathrm{PS}$ & 103.0 & 116 & 143.8 \\
\hline $4.7 \mathrm{k}-\mathrm{L}-50 \% \mathrm{PS}$ & 96.6 & 119 & 147.6 \\
\hline $7.6 \mathrm{k}-\mathrm{S}-50 \% \mathrm{PS}$ & 57.1 & 51 & 63.2 \\
\hline $7.6 \mathrm{k}-\mathrm{S}-40 \% \mathrm{PS}$ & 70.5 & 57 & 70.7 \\
\hline $7.6 \mathrm{k}-\mathrm{L}-50 \% \mathrm{PS}$ & 125.6 & 111 & 137.6 \\
\hline
\end{tabular}




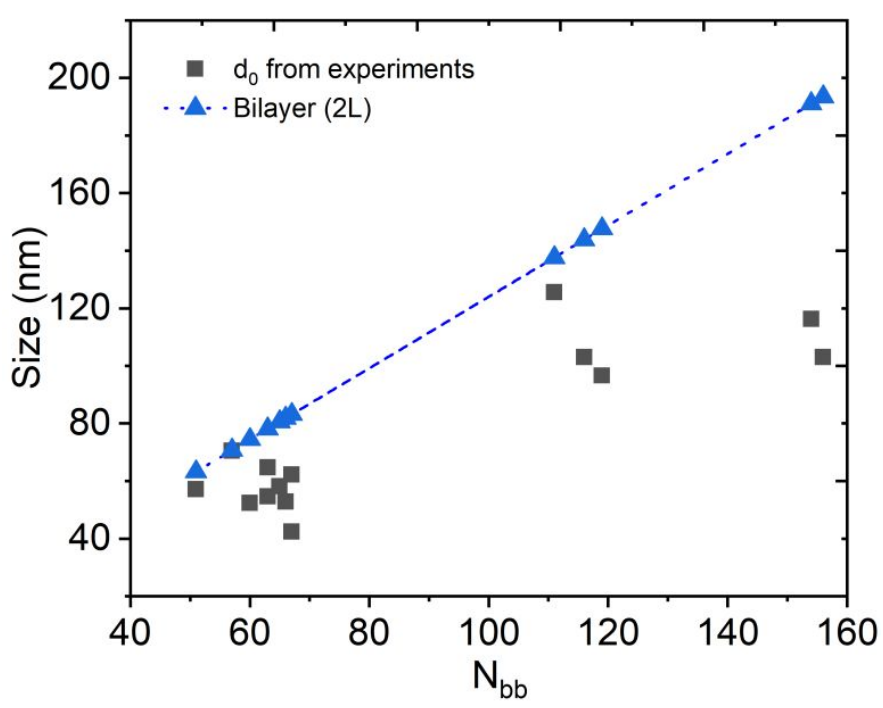

Figure S5 Relationship between domain size and degree of polymerization $N_{b b}$ for lamellar forming PS- $b$-PDMS BBCPs. Upper limit of extended backbones in bi-layer assembly (blue curve) occurs when lamellae size is equal to twice the contour length $\left(2 L=2 * N_{b b} * L_{0}\right)$. All lamellar spacings fall below the limit of extended backbone conformation.

Table S5 Backbone persistence length $\left(l_{\mathrm{p}}\right)$ and contour length $(L)$ for each block of the respective BBCPs samples. The backbone $l_{\mathrm{p}}$ is on the order of side chain size $\left\langle R_{s c}>\right.$ and were calculated according to Reference 2-5. Backbone contour length $L=N_{b b} * L_{0}$, where $L_{0}$ is poly(norbornene) repeat unit length $(0.62 \mathrm{~nm})$. The number of persistence segments per block $\left(L / l_{\mathrm{p}}\right)$ is also calculated.

\begin{tabular}{|c|c|c|c|c|c|c|}
\hline Sample code & $\begin{array}{c}\boldsymbol{l}_{\mathbf{p}}(\mathbf{P D M S}) \\
(\mathbf{n m})\end{array}$ & $\begin{array}{c}\boldsymbol{L}(\mathbf{P D M S}) \\
(\mathbf{n m})\end{array}$ & $\left.\boldsymbol{L} / \boldsymbol{l}_{\mathbf{p}} \mathbf{( P D M S}\right)$ & $\begin{array}{c}\boldsymbol{l}_{\mathbf{p}}(\mathbf{P S}) \\
(\mathbf{n m})\end{array}$ & $\begin{array}{c}\boldsymbol{L}(\mathbf{P S}) \\
(\mathbf{n m})\end{array}$ & $\boldsymbol{L} / \boldsymbol{l}_{\mathbf{p}}(\mathbf{P S})$ \\
\hline $3.1 \mathrm{k}-\mathrm{S}-90 \% \mathrm{PS}$ & 4.7 & 3.1 & 0.66 & 3.7 & 39.1 & 10.56 \\
\hline $3.1 \mathrm{k}-\mathrm{S}-80 \% \mathrm{PS}$ & 4.7 & 6.2 & 1.32 & 3.7 & 37.2 & 10.05 \\
\hline $3.1 \mathrm{k}-\mathrm{S}-70 \% \mathrm{PS}$ & 4.7 & 9.3 & 1.98 & 3.7 & 32.2 & 8.71 \\
\hline $3.1 \mathrm{k}-\mathrm{S}-60 \% \mathrm{PS}$ & 4.7 & 12.4 & 2.64 & 3.7 & 28.5 & 7.71 \\
\hline $3.1 \mathrm{k}-\mathrm{S}-50 \% \mathrm{PS}$ & 4.7 & 15.5 & 3.30 & 3.7 & 23.5 & 6.37 \\
\hline $3.1 \mathrm{k}-\mathrm{S}-40 \% \mathrm{PS}$ & 4.7 & 18.6 & 3.96 & 3.7 & 18.6 & 5.03 \\
\hline $3.1 \mathrm{k}-\mathrm{S}-30 \% \mathrm{PS}$ & 4.7 & 21.1 & 4.49 & 3.7 & 13.6 & 3.69 \\
\hline $3.1 \mathrm{k}-\mathrm{S}-20 \% \mathrm{PS}$ & 4.7 & 29.8 & 6.34 & 3.7 & 11.2 & 3.02 \\
\hline $3.1 \mathrm{k}-\mathrm{S}-10 \% \mathrm{PS}$ & 4.7 & 32.2 & 6.85 & 3.7 & 5.6 & 1.51 \\
\hline $3.1 \mathrm{k}-\mathrm{L}-90 \% \mathrm{PS}$ & 4.7 & 8.1 & 1.72 & 3.7 & 114.1 & 30.80 \\
\hline $3.1 \mathrm{k}-\mathrm{L}-80 \% \mathrm{PS}$ & 4.7 & 13.6 & 2.89 & 3.7 & 84.9 & 22.90 \\
\hline $3.1 \mathrm{k}-\mathrm{L}-70 \% \mathrm{PS}$ & 4.7 & 20.5 & 4.36 & 3.7 & 73.8 & 19.90 \\
\hline $3.1 \mathrm{k}-\mathrm{L}-60 \% \mathrm{PS}$ & 4.7 & 29.1 & 6.19 & 3.7 & 66.9 & 18.10 \\
\hline $3.1 \mathrm{k}-\mathrm{L}-50 \% \mathrm{PS}$ & 4.7 & 37.8 & 8.04 & 3.7 & 58.9 & 15.92 \\
\hline
\end{tabular}




\begin{tabular}{|c|c|c|c|c|c|c|}
\hline $3.1 \mathrm{k}-\mathrm{L}-40 \% \mathrm{PS}$ & 4.7 & 47.1 & 10.02 & 3.7 & 48.4 & 13.07 \\
\hline $3.1 \mathrm{k}-\mathrm{L}-30 \% \mathrm{PS}$ & 4.7 & 42.8 & 9.11 & 3.7 & 27.9 & 7.54 \\
\hline $3.1 \mathrm{k}-\mathrm{L}-20 \% \mathrm{PS}$ & 4.7 & 48.4 & 10.30 & 3.7 & 18.6 & 5.03 \\
\hline $3.1 \mathrm{k}-\mathrm{L}-10 \% \mathrm{PS}$ & 4.7 & 53.3 & 11.34 & 3.7 & 9.3 & 2.51 \\
\hline $4.7 \mathrm{k}-\mathrm{S}-90 \% \mathrm{PS}$ & 4.7 & 4.3 & 0.91 & 4.5 & 41.5 & 9.23 \\
\hline $4.7 \mathrm{k}-\mathrm{S}-80 \% \mathrm{PS}$ & 4.7 & 8.7 & 1.85 & 4.5 & 35.3 & 7.85 \\
\hline $4.7 \mathrm{k}-\mathrm{S}-70 \% \mathrm{PS}$ & 4.7 & 13.6 & 2.89 & 4.5 & 32.9 & 7.30 \\
\hline $4.7 \mathrm{k}-\mathrm{S}-60 \% \mathrm{PS}$ & 4.7 & 15.5 & 3.30 & 4.5 & 23.6 & 5.23 \\
\hline $4.7 \mathrm{k}-\mathrm{S}-50 \% \mathrm{PS}$ & 4.7 & 20.5 & 4.36 & 4.5 & 21.1 & 4.68 \\
\hline $4.7 \mathrm{k}-\mathrm{S}-40 \% \mathrm{PS}$ & 4.7 & 24.2 & 5.15 & 4.5 & 16.1 & 3.58 \\
\hline $4.7 \mathrm{k}-\mathrm{S}-30 \% \mathrm{PS}$ & 4.7 & 28.5 & 6.06 & 4.5 & 11.8 & 2.62 \\
\hline $4.7 \mathrm{k}-\mathrm{S}-20 \% \mathrm{PS}$ & 4.7 & 29.1 & 6.19 & 4.5 & 7.4 & 1.65 \\
\hline $4.7 \mathrm{k}-\mathrm{S}-10 \% \mathrm{PS}$ & 4.7 & 35.9 & 7.64 & 4.5 & 3.7 & 0.83 \\
\hline 4.7k-L-90\%PS & 4.7 & 6.8 & 1.45 & 4.5 & 58.3 & 12.95 \\
\hline $4.7 \mathrm{k}-\mathrm{L}-80 \% \mathrm{PS}$ & 4.7 & 13.0 & 2.77 & 4.5 & 53.3 & 11.85 \\
\hline $4.7 \mathrm{k}-\mathrm{L}-70 \% \mathrm{PS}$ & 4.7 & 19.8 & 4.21 & 4.5 & 47.7 & 10.61 \\
\hline $4.7 \mathrm{k}-\mathrm{L}-60 \% \mathrm{PS}$ & 4.7 & 28.5 & 6.06 & 4.5 & 43.4 & 9.64 \\
\hline $4.7 \mathrm{k}-\mathrm{L}-50 \% \mathrm{PS}$ & 4.7 & 36.6 & 7.79 & 4.5 & 37.2 & 8.27 \\
\hline $4.7 \mathrm{k}-\mathrm{L}-40 \% \mathrm{PS}$ & 4.7 & 43.4 & 9.23 & 4.5 & 29.8 & 6.61 \\
\hline $4.7 \mathrm{k}-\mathrm{L}-30 \% \mathrm{PS}$ & 4.7 & 47.7 & 10.15 & 4.5 & 21.1 & 4.68 \\
\hline $4.7 \mathrm{k}-\mathrm{L}-20 \% \mathrm{PS}$ & 4.7 & 47.1 & 10.02 & 4.5 & 11.8 & 2.62 \\
\hline $4.7 \mathrm{k}-\mathrm{L}-10 \% \mathrm{PS}$ & 4.7 & 51.5 & 10.96 & 4.5 & 6.2 & 1.38 \\
\hline $7.6 \mathrm{k}-\mathrm{S}-90 \% \mathrm{PS}$ & 4.7 & 4.3 & 0.91 & 5.7 & 26.0 & 4.57 \\
\hline $7.6 \mathrm{k}-\mathrm{S}-80 \% \mathrm{PS}$ & 4.7 & 8.7 & 1.85 & 5.7 & 21.1 & 3.70 \\
\hline $7.6 \mathrm{k}-\mathrm{S}-70 \% \mathrm{PS}$ & 4.7 & 12.4 & 2.64 & 5.7 & 18.0 & 3.15 \\
\hline $7.6 \mathrm{k}-\mathrm{S}-60 \% \mathrm{PS}$ & 4.7 & 16.7 & 3.55 & 5.7 & 15.5 & 2.72 \\
\hline $7.6 \mathrm{k}-\mathrm{S}-50 \% \mathrm{PS}$ & 4.7 & 19.2 & 4.09 & 5.7 & 12.4 & 2.17 \\
\hline $7.6 \mathrm{k}-\mathrm{S}-40 \% \mathrm{PS}$ & 4.7 & 24.8 & 5.28 & 5.7 & 10.5 & 1.85 \\
\hline $7.6 \mathrm{k}-\mathrm{S}-30 \% \mathrm{PS}$ & 4.7 & 28.5 & 6.06 & 5.7 & 7.4 & 1.30 \\
\hline $7.6 \mathrm{k}-\mathrm{S}-20 \% \mathrm{PS}$ & 4.7 & 29.7 & 6.32 & 5.7 & 4.3 & 0.76 \\
\hline $7.6 \mathrm{k}-\mathrm{S}-10 \% \mathrm{PS}$ & 4.7 & 31.0 & 6.60 & 5.7 & 2.5 & 0.43 \\
\hline 7.6k-L-90\%PS & 4.7 & 6.8 & 1.45 & 5.7 & 37.2 & 6.53 \\
\hline $7.6 \mathrm{k}-\mathrm{L}-80 \% \mathrm{PS}$ & 4.7 & 14.3 & 3.04 & 5.7 & 35.9 & 6.31 \\
\hline $7.6 \mathrm{k}-\mathrm{L}-70 \% \mathrm{PS}$ & 4.7 & 21.7 & 4.62 & 5.7 & 32.2 & 5.65 \\
\hline $7.6 \mathrm{k}-\mathrm{L}-60 \% \mathrm{PS}$ & 4.7 & 30.4 & 6.47 & 5.7 & 29.1 & 5.11 \\
\hline $7.6 \mathrm{k}-\mathrm{L}-50 \% \mathrm{PS}$ & 4.7 & 42.2 & 8.98 & 5.7 & 26.7 & 4.68 \\
\hline $7.6 \mathrm{k}-\mathrm{L}-40 \% \mathrm{PS}$ & 4.7 & 52.7 & 11.21 & 5.7 & 21.7 & 3.81 \\
\hline $7.6 \mathrm{k}-\mathrm{L}-30 \% \mathrm{PS}$ & 4.7 & 59.5 & 12.66 & 5.7 & 16.1 & 2.83 \\
\hline $7.6 \mathrm{k}-\mathrm{L}-20 \% \mathrm{PS}$ & 4.7 & 57.0 & 12.13 & 5.7 & 9.3 & 1.63 \\
\hline $7.6 \mathrm{k}-\mathrm{L}-10 \% \mathrm{PS}$ & 4.7 & 54.6 & 11.62 & 5.7 & 3.72 & 0.65 \\
\hline
\end{tabular}



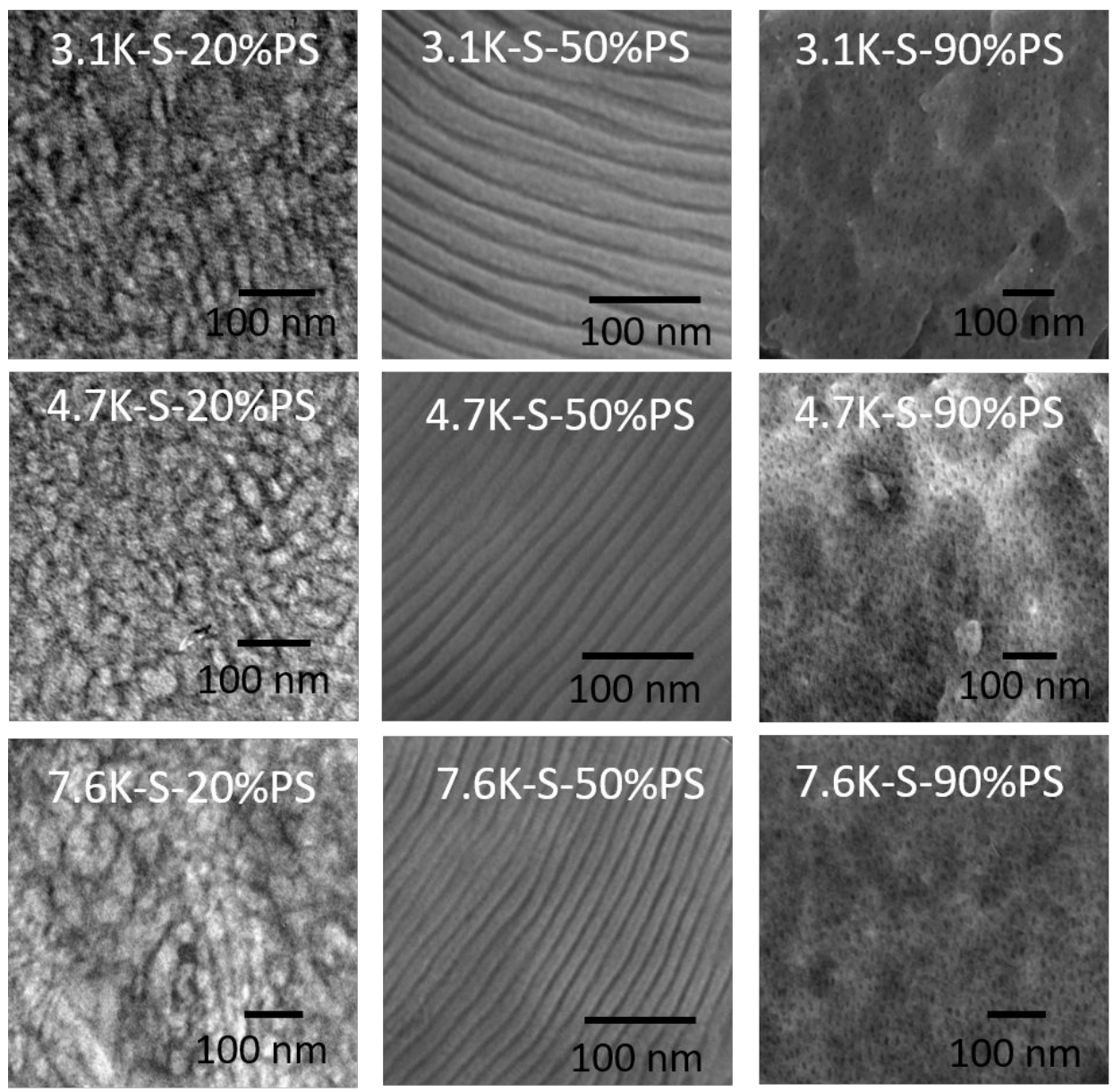

Figure S6 Additional TEM images of PS- $b$-PDMS BBCPs with short backbones at various side chain lengths and volume fractions. 

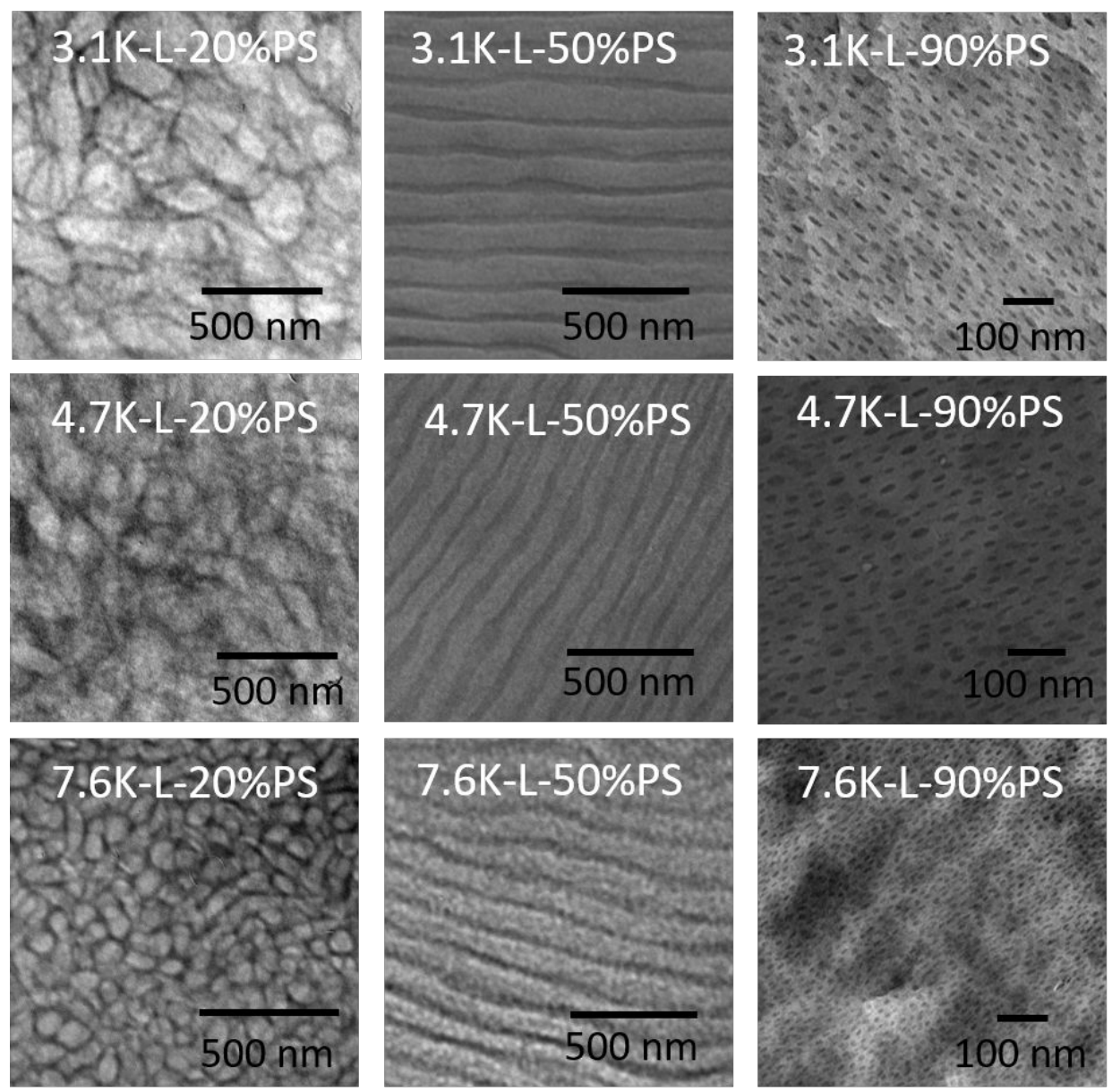

Figure S7 Additional TEM images of PS- $b$-PDMS BBCPs with longer backbones at various side chain lengths and volume fractions.
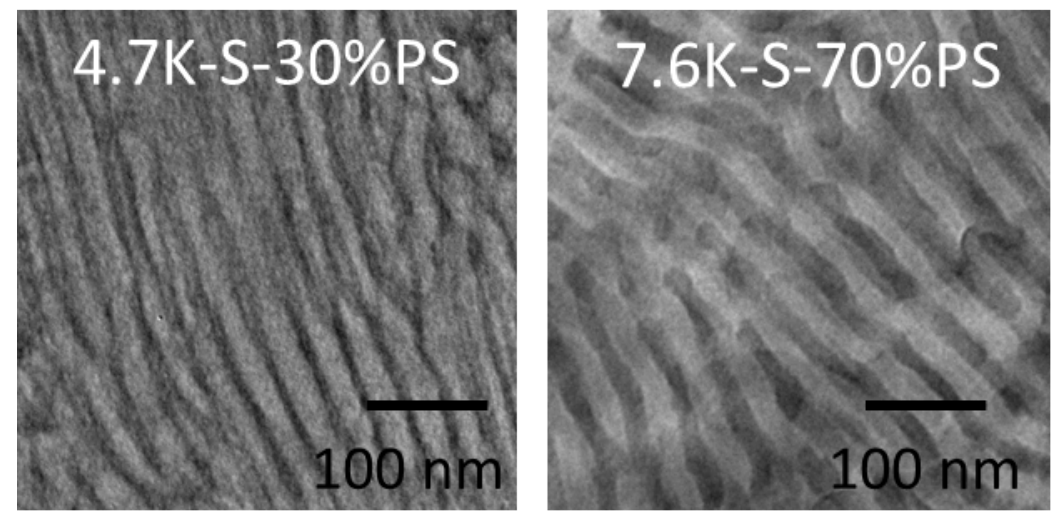

Figure S8 TEM images of samples with cylinder morphology showing in-plane cylinders ("lying down" orientation). 


\section{Calculation of $\left\langle\boldsymbol{R}_{s c}>\right.$ according to packing length concept.}

The side chain size $<R_{s c}>$ is the end-to-end distance of the chain. We determine the size based on the packing length concept described by Fetters et al. The packing length $p$ is a quantity that describes the number of given chains in a volume of melt. From $p$, a normalized size $<R^{2}>/ M$ can be determined. The size of the side chains is estimated based on the reported values of $\left\langle R^{2}>/ M\right.$ from Ref. 2 and the $M_{\mathrm{n}}$ of the side chain macromonomers.

For PS, $<R^{2}>/ M_{\mathrm{n}}=0.434 \AA^{2} \mathrm{~mol} \mathrm{~g}^{-1}$, and $M_{\mathrm{n}}=3100,4700$ and $7600 \mathrm{~g} \mathrm{~mol}^{-1}$. Therefore $<R_{s c}>=$ $3.7,4.5,5.7 \mathrm{~nm}$, respectively.

For PDMS, $<R^{2}>/ M \mathrm{n}=0.457 \AA^{2} \mathrm{~mol} \mathrm{~g}^{-1}$, and $M_{\mathrm{n}}=4800 \mathrm{~g} \mathrm{~mol}^{-1}$. Therefore $<R_{s c}>=4.7 \mathrm{~nm}$

\section{References}

1. Fei, H.; Yavitt, B. M.; Kopanati, G.; Watkins, J. J. Effect of Side Chain and Backbone Length on Lamellar Spacing in Polystyrene-Block-Poly(Dimethyl Siloxane) Brush Block Copolymers. J. Polym. Sci. Part B Polym. Phys. 2019, 57 (11), 691-699.

2. Fetters L. J.; Lohse D. J.; Richter D.; Witten T. A.; Zirkel, A. Connection between Polymer Molecular Weight, Density, Chain Dimensions, and Melt Viscoelastic Properties. Macromolecules 1994, 27, (17), 4639-4647.

3. Fetters L. J.; Lohse D. J.; Milner, S. T. Packing Length Influence in Linear Polymer Melts on the Entanglement, Critical, and Reptation Molecular Weight. Macromolecules 1999, 32, 6847-6851.

4. Milner, S. T. Predicting the tube diameter in melts and solutions. Macromolecules 2005. 38(11), 4929-4939.

5. Paturej, J.; Sheiko, S. S.; Panyukov, S.; Rubinstein, M. Molecular Structure of Bottlebrush Polymers in Melts. Sci. Adv. 2016, 2 (11), e1601478. 